\title{
A Note on Style
}

I used the Library of Congress transliteration system for Russian, Ukrainian, and Belorussian, suppressing soft signs in proper names and with the usual exceptions for well-known names such as Trotsky. The cast of characters and places in this book covers dozens of languages, and it would be impossible to accurately name all non-Russians in their native languages. Therefore, I have used Russian names throughout for individuals' and place names, with the sole exception of several well-known Ukrainians. I have also used the contemporary place names rather than the emerging new ones, such as Kirgizia not Kyrgyzstan. 

The Affirmative Action Empire 
